\title{
Massive coronary artery air embolism due to an unusual cause
}

\author{
IOANNIS TZIATZIOS, MATTHAIOS DIDAGELOS, STEFANOS VOTSIS, \\ GEORGIOS TZIATZIOS, STAVROS HADJIMILTIADES*
}

\author{
First Department of Cardiology, AHEPA Hospital, Aristotle University, Thessaloniki, Greece \\ *Corresponding author: Stavros Hadjimiltiades, MD, PhD, FACC; First Department of Cardiology, AHEPA Hospital, Aristotle University, \\ Stilponos Kyriakidi 1, 54636 Thessaloniki, Greece; Phone: +30 2313 304833; Fax: +30 2310 994673; E-mail: stavros@otenet.gr
}

(Received: October 10, 2017; Accepted: March 5, 2018)

\begin{abstract}
A 46-year-old man underwent angioplasty of a restenotic bifurcation lesion of the circumflex artery. The procedure required repeated balloon exchanges and during the last balloon inflation, no balloon was visualized. A test injection revealed a massive coronary air embolism due to expulsion of air that had accumulated in the guiding catheter shaft. The patient was rapidly resuscitated from electromechanical dissociation with intracoronary injection of adrenaline and atropine and forceful intracoronary saline injections. Inspection of the balloon revealed a defect and scratch marks at the junction of the wire part and shaft of the monorail balloon, a location that places the air leakage inside the guiding catheter. This is the first report of massive intracoronary air embolism due to an undetectable damage to the shaft of a balloon angioplasty catheter. Recognition of the problem and immediate intervention is vital in limiting the duration of cardiac dysfunction.
\end{abstract}

Keywords: air embolism, complications, angiography, angioplasty, balloon shaft defect

\section{Introduction}

Coronary air embolism is an uncommon but wellrecognized complication of coronary catheterization that occurs in $0.1 \%-0.3 \%$ of cases, most frequently in angioplasty cases [1-4]. Most often, air embolism occurs due to inadequate aspiration and flushing of catheters [5]. Other possible mechanisms of coronary air embolism that have been described during coronary artery angioplasty are balloon rupture, intraguide catheter suction of air during balloon catheter or guide wire exchanges, structural defects of the equipment, and continuous negative suction of special balloon catheters $[3,5]$.

\section{Case Report}

A 46-year-old man with a family history of premature coronary artery disease, dyslipidemia and two previous angioplasties for restenosis of a bifurcation lesion of a dominant left circumflex coronary artery (LCx), was admitted because of new onset effort angina 4 months post his last angioplasty. The new coronary angiogram that was performed demonstrated restenosis proximal to and at the bifurcation site of the LCx (Fig. IA, Video), a known chronic occlusion of a small right coronary artery and good systolic left ventricular function with no segmental abnormalities.

An angioplasty was performed with an EBU 3.5 6F guiding catheter (Launcher, Medtronic, MA, USA) by kissing balloon inflations with a $3 / 20-\mathrm{mm}$ balloon (Artimes, BrosMed, KM, Netherlands) in the continuation of the LCx and a 3.5/20-mm balloon (Maverick, Boston Scientific, MA, USA) in the main vessel, over two cougar XT guide wires (Medtronic, MA, USA). A 3.5/ 9-mm stent (Resolute Integrity, Medtronic, MA, USA) was deployed just proximal to the bifurcation followed by sequential and kissing balloon inflations with the previously used balloon catheters at the bifurcation site.

An additional inflation was deemed necessary and the previously used 3/20 balloon (Artimes, BrosMed) was prepared again by applying negative pressure and was inserted and advanced to the LCx for the third time. The plunger of the indeflator was advanced, but the

This is an open-access article distributed under the terms of the Creative Commons Attribution-NonCommercial 4.0 International License, which permits unrestricted use, distribution, and reproduction in any medium for non-commercial purposes, provided the original author and source are credited, a link to the CC License is provided, and changes - if any - are indicated. 


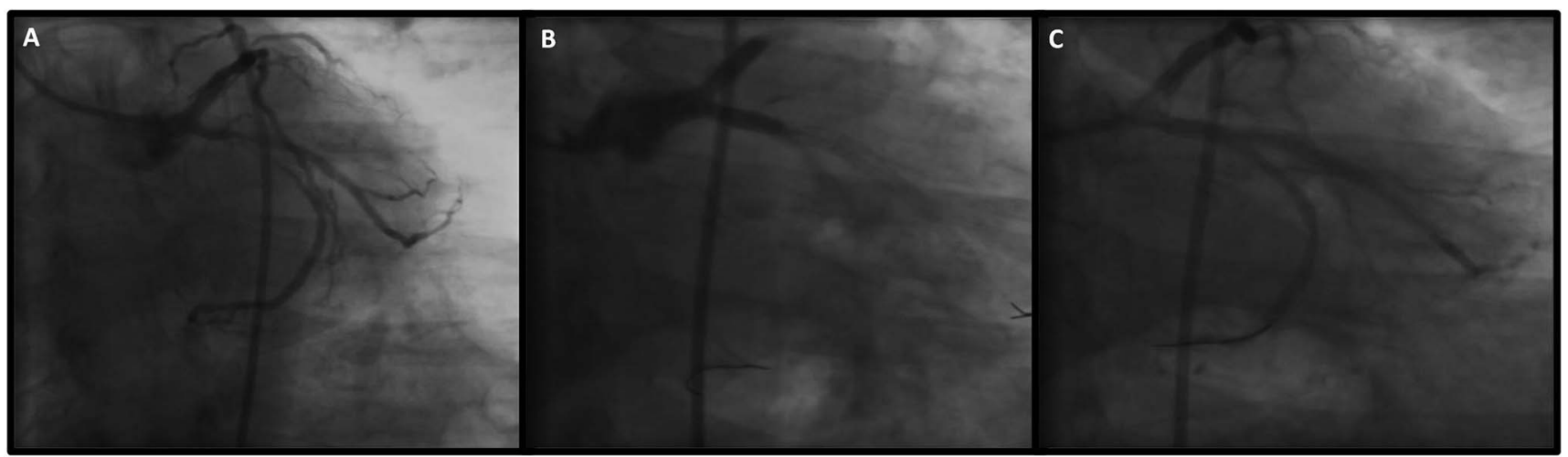

Fig. 1. (A) Initial left coronary angiogram demonstrating the restenotic bifurcation lesion of the dominant LCx. (B) Left coronary angiogram after massive air embolism. (C) Final angiogram demonstrating complete patency of the arteries

balloon was not visualized and the first thought was that the balloon was not well prepared and was inflated with air or saline. A small test injection was not informative and was followed by ST segment elevation noticed on the ECG monitor while the patient started complaining of precordial chest pain. On a second injection, the angiographic picture was that of complete occlusion of the proximal left anterior descending artery and the proximal dominant LCx with no distal vessel visualization. The first impression was that of massive air embolism, but the injection of thrombotic material was also a possibility (Fig. 1B, Video). There was a moment of uncertainty as to the source of such a large amount of air distributed to the whole left coronary artery while the balloon was located at the distal part of the LCx.

The patient collapsed with electromechanical dissociation. We administered $1 \mathrm{mg}$ of adrenaline and $1 \mathrm{mg}$ of atropine intracoronary followed by forceful injections of warm saline. The patient was ventilated at $100 \%$ oxygen with a mask. The period of circulatory collapse lasted less than $3 \mathrm{~min}$ and then suddenly the pressure jumped to $240 / 160 \mathrm{mmHg}$ and the heart rate to 150 beats per minute (bpm). About $10 \mathrm{~min}$ later, the arterial blood pressure was $70 / 50 \mathrm{mmHg}$, the heart rate $110 \mathrm{bpm}$, and the patient required intravenous dopamine for hemodynamic support. The final angiographic evaluation showed a satisfactory angiographic result with TIMI-3 flow in the whole left coronary artery (Fig. 1C, Video). An echocardiographic evaluation demonstrated good left ventricular function with no segmental abnormalities.

Inspection of the balloon catheter under water demonstrated that the air was escaping during inflation of the balloon at the transition bonding joint, where the wire shaft meets the hard shaft of the catheter (Video).

Post-procedurally, there was a mild increase of the cardiac enzymes and the ECG showed negative T waves in leads Vl-V6, which $12 \mathrm{~h}$ later reverted to positive. The patient required intravenous dopamine infusion for several hours due to persistent low blood pressure, despite the good left ventricular function. He was discharged 2 days later in hemodynamically stable condition.

\section{Discussion}

The signs and symptoms associated with air embolism result from the interruption of blood flow within the artery [3]. The clinical presentation of coronary air embolism includes chest pain, hypotension, life-threatening arrhythmias, ECG changes of myocardial ischemia, and even cardiac arrest [2-5]. The severity of symptoms is proportional to the introduced air's volume, the number of affected vessels, and the extent of the baseline myocardial dysfunction [3]. The trapped air interrupts the blood flow producing ischemic dysfunction of the myocardium and due to the surface tension of the distorted bubble with a larger radius proximally and smaller distally, very large pressure gradients are required to move the air through the capillaries. In addition, endothelial dysfunction of the microcirculation appears, as the air embolus divides into smaller bubbles and travels through capillaries damaging the endothelium through mechanical and humoral and cellular immune mechanisms $[2,3]$.

The main treatment objective is to expel air from the coronary circulation as fast as possible. When the blood pressure falls and bradycardia and electromechanical dissociation appear, intracoronary administration of adrenaline and atropine seems to be the best and readily available solution. This action increases the coronary blood flow and causes division of the embolus through vasospasm $[2,3]$. At the same time, vigorous and repetitive injection of saline into the coronary arteries increases the local pressure and may help in advancing the air lock. Other mechanical measures that have been described include air embolus aspiration using thrombectomy catheters or over-thewire balloons [1,6]. Simultaneously, inhalation of $100 \%$ oxygen accelerates air embolus shrinkage and 
absorption considering that the main gas in air is nitrogen. Finally, intracoronary administration of adenosine, calcium channel blockers, or nitrates may be useful after the patient's hemodynamic stabilization, as they can deal with the slow-flow phenomenon [6].

The knowledge of all the possible avenues of air introduction is very important during an interventional procedure, not only for prevention but also for the rapid recognition and confident approach in case air embolism happens. In our case, the initial diagnosis and mechanism of the massive air embolism was not obvious, but the angiographic picture was highly suggestive of air embolism. As it was described, we tested the balloon catheter under water by inflating the balloon with air. This did not reveal a balloon defect but to our surprise revealed air leakage at the junction of the wire lumen with the stiffer part of the shaft of this monorail balloon catheter (Video). The scanning of the catheter shaft performed by the company's lab revealed a crack with tidy edges at the site of the leakage and an obvious scratch extending all the way to the balloon. It should be mentioned that the shaft bursting pressure is much higher than the bursting balloon pressure. Most probably, the defect was the result of friction with the wires and the wall of the guiding catheter, considering that the balloon had been retrieved and advanced three times with an additional balloon and wire in place.

Apparently, air was introduced into the indeflator system through the crack of the shaft, when the balloon catheter was left on suction outside the body of the patient. With the balloon in the distal LCx, the attempt to inflate the balloon resulted in air leaking into the lumen of the guiding catheter, forming a column of air that was subsequently injected. To our knowledge, this is the first report of massive intracoronary air embolism due to a shaft defect of a balloon angioplasty catheter. Apparently, a series of events starting with the friction of catheters and wires in a confined space resulted in a defect of the shaft, which would have been inconsequential, if the balloon catheter was not left on suction in ambient air.

\section{Conclusions}

In conclusion, we describe an unusual point of air entry, the shaft of the balloon catheter, with the potential of causing massive coronary air embolism during coronary angioplasty not during the inflation of the balloon, but during the subsequent contrast injection. It is advisable that in case the balloon of the coronary catheter is not visualized while being inflated, no injections should be made, the balloon catheter should be removed and the guiding catheter should be well aspirated. Irrespective of its causes, massive coronary air embolism remains a frightening complication during invasive procedures and requires prompt and aggressive treatment.

$$
\text { *** }
$$

Funding sources: No financial support was received for this study.

Authors' contribution: SH, IT, and MD: interventional procedure and final approval of the version to be published. SV and GT: literature review, drafting of the manuscript, and final approval of the version to be published. All authors had full access to all data in the study and take responsibility for the integrity of the data and the accuracy of the data analysis.

Conflict of interest: The authors declare no conflict of interest.

\section{References}

1. Patterson MS, Kiemeneij F: Coronary air embolism treated with aspiration catheter. Heart 91, e36 (2005)

2. Khan M, Schmidt DH, Bajwa T, Shalev Y: Coronary air embolism: Incidence, severity, and suggested approaches to treatment. Cathet Cardiovasc Diagn 36, 313-318 (1995)

3. Dib J, Boyle AJ, Chan M, Resar JR: Coronary air embolism: A case report and review of the literature. Catheter Cardiovasc Interv 68, 897-900 (2006)

4. Yang HS, Kim JY, Moon SW, Jung JH, Kim DH, Oh YS, Jo JH, Jeong $\mathrm{MH}$ : A case of coronary air embolism of the left coronary arteries that manifested as cardiogenic shock. Korean Circ J 37, 334-336 (2007)

5. Park CB, Hwang HJ, Cho JM, Jo BH, Kim CJ: Massive right coronary air embolism in the right coronary artery during left coronary angiography: A case report. Exp Ther Med 5, 1073-1074 (2013)

6. Yew KL, Razali F: Massive coronary air embolism successfully treated with intracoronary catheter aspiration and intracoronary adenosine. Int J Cardiol 188, 56-57 (2015)

\section{Electronic Supplementary Material (ESM)}

Electronic Supplementary Material (ESM) associated with this article can be found at the website www.akademiai. com/doi/suppl/10.1556/1646.10.2018.16

\section{ESM1}

Video. Characteristic angiographic views from the procedure and ex-vivo inspection of the balloon catheter 